\title{
Numerical simulation of tool/abradable material interaction experiments
}

A. Batailly, Laboratoire de dynamique des structures et vibrations, université McGill, Québec M. Cuny, École Nationale d'Ingénieurs de Metz, France

M. Legrand, Laboratoire de dynamique des structures et vibrations, université McGill, Québec

\section{Abstract}

Applying abradable coating on the casing of turbomachines has been widely recognized as a robust solution advantageously combining the adjustment of operating clearances with the reduction of potential non-repairable damages. Thus, the modeling of this material is a growing field of investigation. Based on the numerical strategy proposed and detailed in previous publication by the same authors, the present study aims at capturing the mechanical behavior of abradable coating in the context of high speed interaction with a rigid tool. The plastic law given is first enriched in order to take into account strain rate dependence. The sensitivity of the model regarding its main numerical parameters is assessed and highlights the role of each of these parameters. The calibration of numerical results with respect to experimental results lead to very satisfying results that confirm that the proposed strategy is well-suited for the modeling of abradable coating. Finally, the newly developped viscoplastic law is applied to a $3 D$ rotor/stator interaction case to determine the criticity of strain rate dependence in the case of blade/casing contact.

\section{Introduction}

The minimization of parasitic leakage flows between each stage of the compressor is a key feature of the optimization of the power efficiency of turbomachines. Accordingly, designers focus on the reduction of the clearance between rotating parts and structural interaction through direct contact between these components is thus more likely to occur. Because these interactions may lead to potentially dangerous phenomena, their understanding and control is a large field of investigation involving both numericists $[1,2]$ and experimenters [3].

Applying abradable coating on the casing [4] has been widely recognized as a robust solution advantageously combining the adjustment of operating clearances with the reduction of potential non-repairable damage. This material should be soft enough not to lead to significant wear of the blades and sufficiently resilient to stand very high temperatures and high-speed gas flows with inherent solid particles [5]. 
Recent numerical developments [6, 7] focused on the modeling of the abradable coating in the context of full 3D contact simulations on one stage of an aircraft engine compressor and obtained results were consistent with physical considerations such as structural resonance. In [6], abradable coating is modeled with a mono-dimensional plastic law for which an accurate calibration may not be easily achieved on full 3D contact simulations due to the complexity of the contact configuration and the inherent costs of the industrial experiments that would be required. A few studies may be found in the literature regarding the identification of mechanical properties of abradable materials [5, 8, 9]. Most of them deal with static or low speed standard scratch tests due to the aforementioned restrictions.

In our study, based on the existing modeling of abradable coating detailed in [6], the focus is made on the numerical simulations of experiments detailed in the first section. These tests considered a tool/abradable material interaction case between abradable material M601 and a tool made of steel 42CrMo4. Abradable material M601 has been widely studied and a significant amount of information may be found in the literature regarding its material properties $[5,10]$.

Through the numerical simulation of these tests, it is expected to calibrate the material parameters of the mono-dimensional plastic law used in [6] in order to achieve more accurate blade-tip/abradable coating interaction simulations.

\section{Vocabulary}

Two different types of interactions with similar names are considered in this study:

1. The tool/abradable material interaction refers to an experiment involving a tool and a projectile with an abradable layer as described in the following section. The part of the tool in contact with the projectile is called the blade and will be referred to as the blade-edge of the tool.

2. The blade-tip/abradable coating interaction refers to what may happen within a turbomachine on a stage where abradable coating is added as a sacrificial layer between the bladed disk and the casing in order to reduce clearances. In that case, the blade defines an elementary cyclic sector of the bladed disk involved in the interaction. The part of the blade in contact with the abradable coating will be referred to as the blade-tip.

Because blade-tip/abradable coating interaction involves very large and expensive industrial structures, its experimental study is extremely costful and it is hoped it may be simulated with tool/abradable material interaction described in the following section. 


\section{Experimental set-up}

The tool/abradable coating contact phenomenon is studied thanks to an experimental device specially designed to measure contact forces during the interaction depicted in Fig. 1. The device is mainly made of a pneumatic gun to launch a projectile which velocity may reach up to $500 \mathrm{~m} \cdot \mathrm{s}^{-1}$. This projectile supports the abradable material sample. It is guided along a launch tube and interacts with a tool representing the blade-edge pictured in Fig. 1. The main purpose of these experiments is to characterize the material behavior of the abrad-

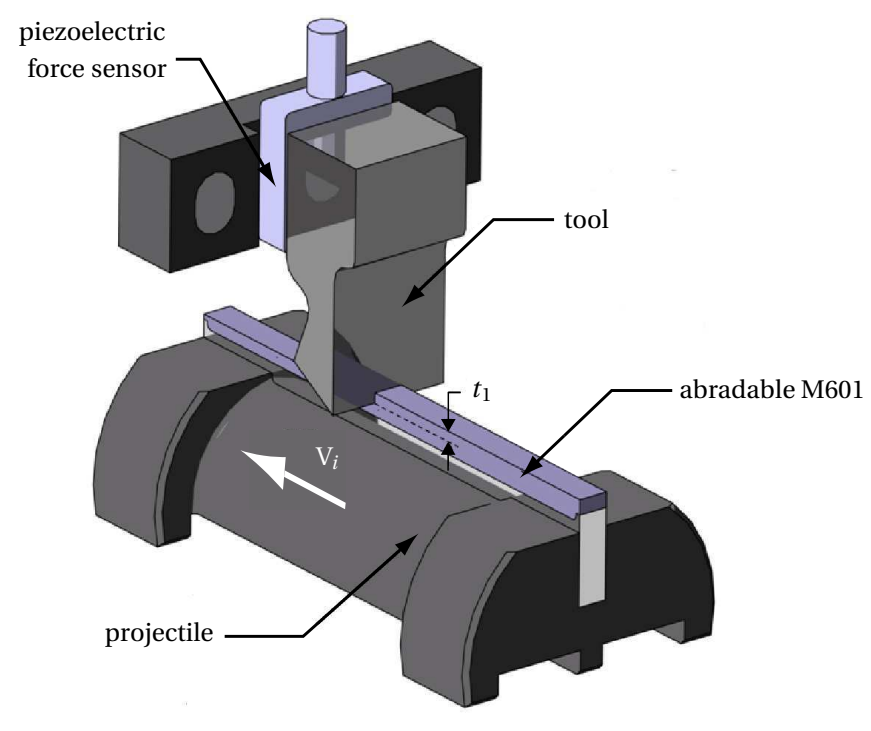

Figure 1 - Zoom over the tool of the test bench and the projectile.

able coating M601 through the measurement of the axial interaction reaction, regardless of the tool dynamics, to feed numerical models such as the one presented in this work.

Obviously, many parameters have an influence on the wear law such as the interaction velocity, the incursion depth, the shape of the blade-edge of the tool, the tool material, etc. However, as a first approach, only the first two parameters were investigated experimentally. The tool shape and material did not vary during the experiments, its blade-edge was regularly sharpened to maintain its radius to $25 \cdot 10^{-3} \mathrm{~mm}$. The tool was made of steel $42 \mathrm{CrMo} 4$ which differs from the usual material of a compressor blade. The choice of this material was made for practical reasons and in agreement with the hypothesis that the tool material has a negligible influence on the interaction forces in comparison with the ones of the velocity and the incursion depth. The same assumption has been made during the modelisation. Orthogonal cutting experiments on abradable material M601 were performed for a range of interaction velocities varying from $60 \mathrm{~m} \cdot \mathrm{s}^{-1}$ to $270 \mathrm{~m} \cdot \mathrm{s}^{-1}$. The theoretical incursion depth was set to $\mathrm{p}=0.2 \mathrm{~mm}$ for all the experiments. Experimental results, depicted in Fig.2, show that the interaction force non-linearly increases with the interaction speed. 


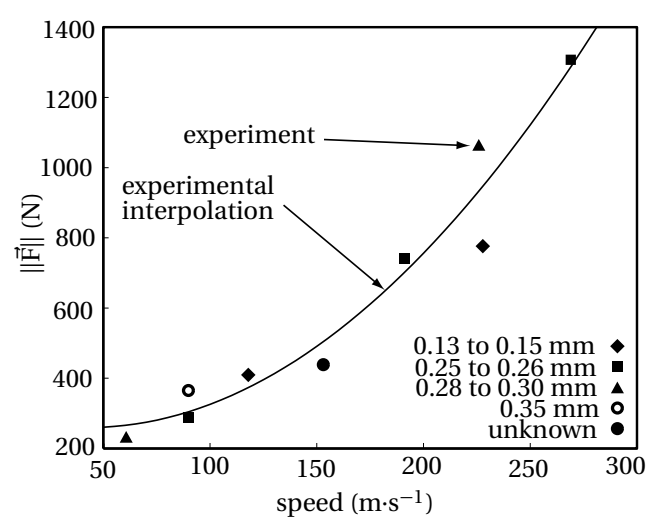

Figure 2 - Experimental observations.

\section{Modeling}

The choice of a generic representation of the tool was made in agreement with the hypothesis that the tool design has a negligible influence on the interaction phenomenon.
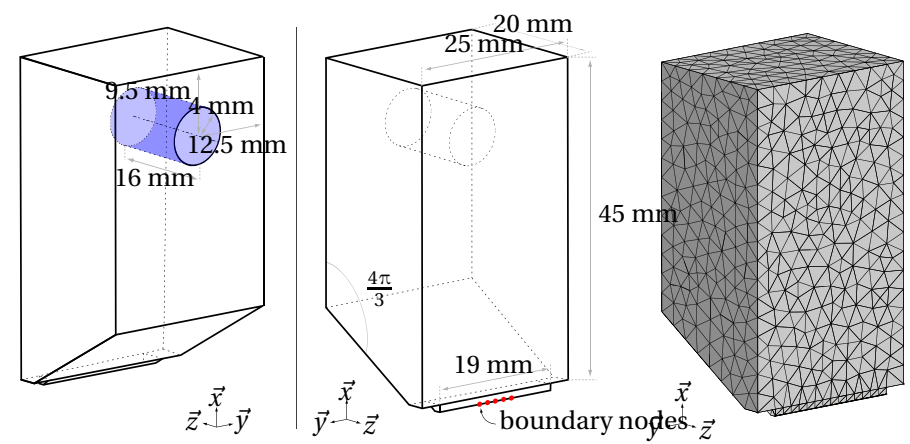

Figure 3 - Mesh and boundary conditions applied on the model of the tool.

The mesh depicted in Fig. 3 is the result of a compromise between the number of nodes and the accuracy of the approximation of its first eigenfrequencies. In total, the mesh is composed of 1,835 nodes and 8,913 linear tetrahedron elements. The tool is clamped on the test bench through an hole made on its back side as depicted in Fig. 3. All the nodes of the mesh on the surface of this hole (pictured in blue) are clamped.

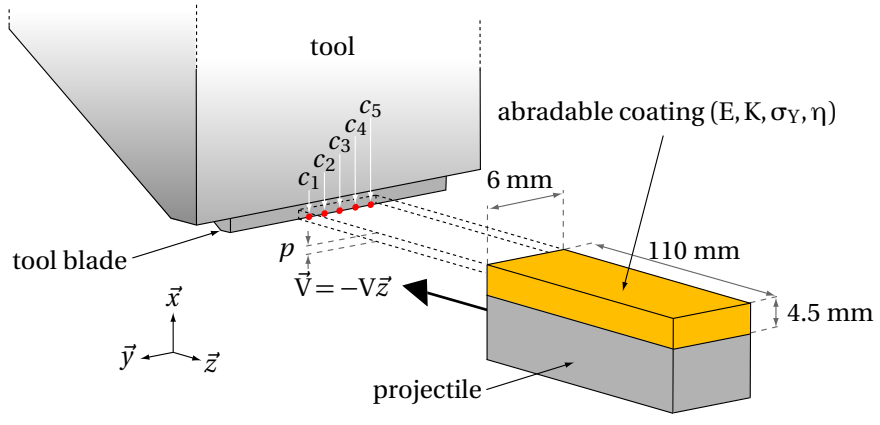

Figure 4-Graphic representation of the numerical simulation.

Fifteen nodes are symmetrically positioned on the edge of the tool blade. Because the width of the projectile is one third of the blade width, only the five middle nodes are considered to manage contact. These five nodes - namely $\left(c_{\mathrm{i}}\right)_{\mathrm{i}=1 . .5}$ and pictured in red in Fig. 3 


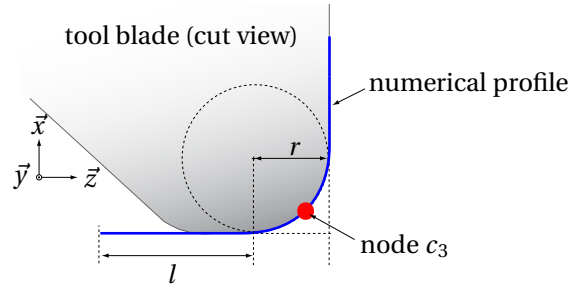

Figure 5 - Zoom on the tool blade: cut view in the $\left(c_{3}, \vec{z}, \vec{x}\right)$ plane.

- correspond to the nodes facing the abradable coating during the interaction as it may be seen in Fig. 4.

The precision of a full finite element model appears as unnecessary in our study and a reduced order model of the tool is created in order to optimize computation times. The Craig-Bampton [11] component mode synthesis method is used, its compatibility with the contact algorithm we use has been previously studied as well as its advantages with respect to other component mode synthesis methods. The final reduced order model of the tool contains 75 degrees of freedom and allows for a very accurate approximation of the 25 first eigenfrequencies of the full finite element model ${ }^{1}$.

Regarding the contact configuration, the abradable coating is placed on a projectile launched by a pneumatic air gun. A simplified representation of this configuration is pictured in Fig. 4 . In order to represent the width of the tool blade in the $\vec{z}$ direction, a numerical profile is used for each contact node as depicted in Fig. 5.

This profile allows for an accurate modeling of the tool blade, considering its width $l^{2}$, and its sharpness $r$. The abradable material is modeled with one-dimensional two-node bar elements depicted in Fig. 6. For the sake of clarity, the relative position of the abradable material and the tool numerical profile for two consecutive time steps is represented in a frame attached to the projectile so that the tool is virtually moving.

Between two consecutive time-steps, the detection of penetrations between the abradable elements and the blade profile allows for the computation of contact efforts. While the abradable elements can only be deformed in the $\vec{x}$ direction, the orientation of the contact effort $\vec{f}_{\mathrm{i}}$ applied on the tool is determined for each abradable element by the normal vector to the numerical at each intersection as depicted in Fig. 6. Accordingly, at each time step, the computed contact effort on each contact node $k,(k=1 . .5)$ is given by: $\overrightarrow{\mathrm{F}}_{\mathrm{k}}=\sum_{\mathrm{i}} \vec{f}_{\mathrm{i}}$. In order to remain consistent with the experiments presented in the first section, only the axial component of the contact effort is considered in the results presented in the paper: the contact force $\mathbf{F}$ of interest is defined as

\footnotetext{
${ }^{1}$ The error between the 10 first eigenfrequencies computed with the reduced order model and with the full finite element model is less than $1 \%$.

${ }^{2} l$ is a numerical parameter that does not influence the computation of the contact force $\mathbf{F}$. Indeed, only the axial component is considered, as detailed in Eq. (1)
} 


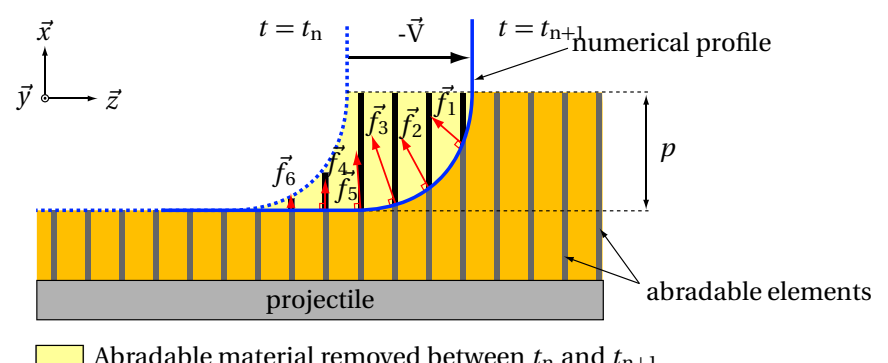

Figure 6 - Numerical profile/abradable material interface.

$$
\mathbf{F}=\sum_{\mathrm{k}} \overrightarrow{\mathrm{F}}_{\mathrm{k}} \cdot \vec{z}
$$

\section{Numerical methods}

The numerical simulation of the tool/abradable material interaction experiments is based on the developments previously described in $[1,6]$. As a consequence they are not fully detailed in this paper.

The explicit time integration scheme relies on the central finite difference method for which the time step is $h=10^{-8} \mathrm{~s}$. Asymptotical convergence of the simulation while diminishing the time step may not be easily shown since simulation diverges for relatively large time steps but one may observe the perfect superimposition of the results for three time steps smaller than $h$ in Fig. 7.

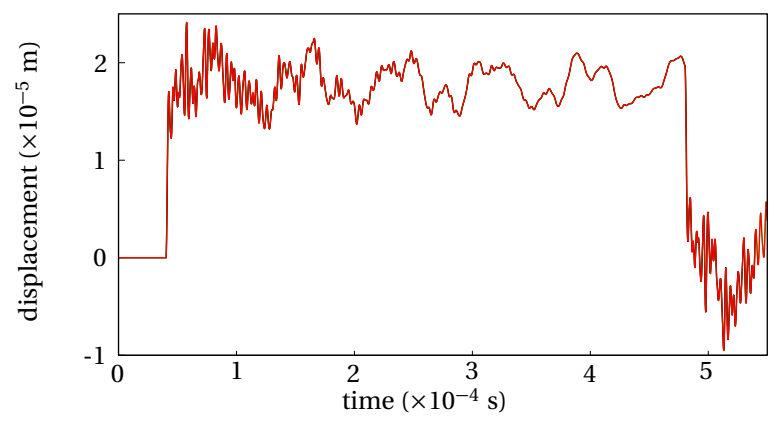

Figure 7 - Displacement of node $c_{3}$ in the $\vec{x}$ direction for different time steps: $h=$ $10^{-8} \mathrm{~s}(-), h=5 \cdot 10^{-9} \mathrm{~s}(-)$ and $h=10^{-9} \mathrm{~s}(-)$. Other parameters are $\|\overrightarrow{\mathrm{V}}\|=200 \mathrm{~m} \cdot \mathrm{s}^{-1}$, $p=0.5 \mathrm{~mm}$.

Regarding the management of abradable material removal, the mono-dimensional plastic law described in [6] is used and adapted to our configuration. Explanations about this adaptation are given in the following section. In order to reach space convergence for all the interaction speeds considered 50,000 abradable elements - which length corresponds to the thickness of the abradable profile - are used along the length of the projectile ${ }^{3}$

\footnotetext{
${ }^{3} 50,000$ abradable elements are used in front of each contact nodes so that the total number of abradable elements is 250,000 .
} 


\section{Plastic law}

The plasticity law used in [6] for the modeling of the abradable material is one-dimensional, strain-rate independent with isotropic hardening. When a quasi-rigid tool is considered, one main drawback of this law is revealed since the simulations of the tool/abradable material interaction show that the contact effort on the tool is independent on the speed of the projectile with abradable material.

This drawback did not clearly appear for blade-tip/abradable coating interaction simulations. Indeed, the wear profiles of the abradable coating varied very much with the rotational velocity of the blade [7]: depending on the rotational velocity, the frequency of the contact force applied on the blade changes and the amplitude of the blade response fluctuates with its rotational velocity as well as the wear level of the abradable coating.

Because the results of the tests clearly highlight the dependency of the contact force on the interaction speed, the plasticity law must be modified and it is proposed to consider a one-dimensional classical viscoplasticity law.

\subsection{One-dimensional plasticity}

The plasticity law detailed in [6] is pictured in Fig. 8(a) and is not fully detailed here for the sake of brevity. The reader may refer to [6] for more details. This law may be summarized using the following equations:

$$
\begin{aligned}
& \sigma=\mathrm{E}\left(\varepsilon-\varepsilon^{\mathrm{p}}\right) \\
& \dot{\varepsilon}^{\mathrm{p}}=\gamma \cdot \operatorname{sign}(\sigma) \\
& \dot{\alpha}=\gamma \\
& f(\sigma, \alpha)=\|\sigma\|-\left(\sigma_{\mathrm{Y}}+\mathrm{K} \cdot \alpha\right) \leq 0 \\
& \gamma \geq 0, f(\sigma, \alpha) \leq 0, \gamma \cdot f(\sigma, \alpha)=0 \\
& \gamma \cdot \dot{f}(\sigma, \alpha)=0(\text { if } f(\sigma, \alpha)=0)
\end{aligned}
$$

where superscript ${ }^{\mathrm{p}}$ stands for plastic. Parameter $\gamma$ stands for the flow rule defined by $\gamma=\dot{\varepsilon}^{\mathrm{vp}}$ and $\alpha$ is usually called internal hardening variable and $\dot{\alpha}=\gamma$. By definition, when a strainrate independent plasticity law is considered, the criterion function $f$ must be negative at all time: $f \leq 0$. This mathematical criterion may be easily graphically interpreted and defines the admissible domain pictured in Fig. 8(a) in which the point $(\varepsilon, \sigma)$ must belong at all time ${ }^{4}$.

\footnotetext{
${ }^{4} \mathrm{~A}$ symmetric domain with respect to the abscissa axis may be considered depending on the type of solicitation of the plastic element.
} 


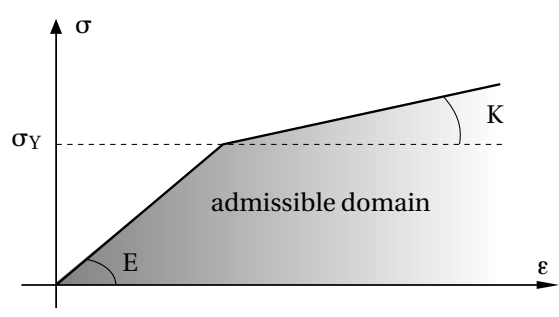

(a) Plasticity

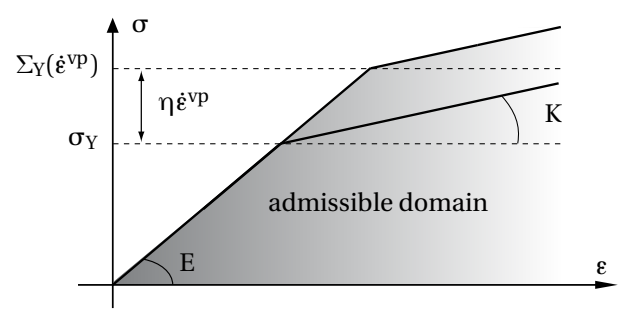

(b) Viscoplasticity

Figure 8 - Constitutive laws controlling the ductility of the abradable.

\subsection{One-dimensional viscoplasticity}

Because of the very high linear speed considered in this study and the level of penetration within the abradable material, strain rate exceeds $\dot{\varepsilon}=10^{3} \mathrm{~s}^{-1}$ which is frequently considered as the threshold after which strain rate cannot be neglected in the plasticity law $[12,13]$.

Moreover, when dealing with structural metals such as titanium and aluminium - which is the main component of abradable material M601 [5] - experiments [14] show that the strain rate strongly affects the yield strength $\sigma_{Y}$ of the material.

It yields that to the contrary of usual plasticity laws, an admissible domain such as the one pictured in Fig. 8(a) may not be defined and the criterion function $f$ defined in Eq. (2) may now be positive. The assumption is made that :

$$
f \geq 0 \Rightarrow f=\sigma_{\mathrm{ex}}=\eta \cdot \dot{\varepsilon}^{\mathrm{vp}}
$$

where $\sigma_{\text {ex }}$ is usually called the extra stress and $\eta$ is a viscous coefficient expressed in Pa.s. A new criterion function $g$ may now be defined: $g(\sigma, \alpha)=f(\sigma, \alpha)+\eta \cdot \dot{\varepsilon}^{\mathrm{vp}}$ which yields: $g(\sigma, \alpha)=\|\sigma\|-\left(\Sigma_{Y}+K \cdot \alpha\right)$. The criterion function $g$ respects the condition $g \leq 0$ thus defining a scalable admissible domain similar to the one presented in the strain-rate independent case and depicted in Fig. 8 (b) with: $\Sigma_{\mathrm{Y}}\left(\dot{\varepsilon}^{\mathrm{vp}}\right)=\sigma_{\mathrm{Y}}+\eta \cdot \dot{\varepsilon}^{\mathrm{vp}}$. In the end, the evolution from the plastic law used in [6] to the proposed viscoplastic law leads to taking into account a variable yield limit depending on the viscoplastic strain-rate $\dot{\varepsilon}^{\mathrm{vp}}$. The computation of the viscoplastic strain-rate $\dot{\varepsilon}^{\mathrm{vp}}$ is the cornerstone of the modifications of the algorithm presented in the following.

\subsection{Algorithm}

The equations developed in this section take into account the specificity of the contact configuration. In particular, an axial stress $\sigma$ within the bar element can only be positive which simplifies, in some degree, the equations.

It is assumed that the state of system at the $n^{\text {th }}$ time step is given: $\sigma_{n}, \varepsilon_{n}, \varepsilon_{n}^{\mathrm{vp}}, \alpha_{\mathrm{n}}$ are known. The state of the system at the next time step is predicted considering a purely elastic 
step:

$$
\begin{aligned}
& \sigma_{\mathrm{n}+1}^{\mathrm{g}}=\mathrm{E}\left(\varepsilon_{\mathrm{n}+1}-\varepsilon_{\mathrm{n}}^{\mathrm{vp}}\right) \\
& \varepsilon_{\mathrm{n}+1}^{\mathrm{vp}, \mathrm{g}}=\varepsilon_{\mathrm{n}}^{\mathrm{vp}} \\
& \alpha_{\mathrm{n}+1}^{\mathrm{g}}=\alpha_{\mathrm{n}} \\
& f_{\mathrm{n}+1}^{\mathrm{g}}=\left\|\sigma_{\mathrm{n}+1}^{\mathrm{g}}\right\|-\left(\sigma_{Y}+K \alpha_{\mathrm{n}}\right)
\end{aligned}
$$

where superscript ${ }^{\mathrm{g}}$ stands for guessed and refers to the predicted values. From the predicted states, two cases are to be considered:

1. $f_{\mathrm{n}+1}^{\mathrm{g}} \leq 0$, then the step is indeed purely elastic and:

$$
\begin{aligned}
& \sigma_{\mathrm{n}+1}=\sigma_{\mathrm{n}+1}^{\mathrm{g}} \\
& \varepsilon_{\mathrm{n}+1}^{\mathrm{vp}}=\varepsilon_{\mathrm{n}}^{\mathrm{vp}} \\
& \alpha_{\mathrm{n}+1}=\alpha_{\mathrm{n}}
\end{aligned}
$$

2. $f_{\mathrm{n}+1}^{\mathrm{g}}>0$, then $f_{\mathrm{n}+1}=\eta \dot{\varepsilon}_{\mathrm{n}+1}^{\mathrm{vp}}$ and viscoplastic strain $\dot{\varepsilon}_{\mathrm{n}+1}^{\mathrm{vp}}$ must be computed:

$$
f_{\mathrm{n}+1}^{\mathrm{g}}>0 \Rightarrow \Delta \gamma>0 \Rightarrow \varepsilon_{\mathrm{n}+1}^{\mathrm{vp}} \neq \varepsilon_{\mathrm{n}}^{\mathrm{vp}} \text { and } \sigma_{\mathrm{n}+1} \neq \sigma_{\mathrm{n}}
$$

where $\Delta \gamma=\int_{t_{\mathrm{n}}}^{t_{\mathrm{n}+1}} \gamma d t$. Coming back to the definition of $\sigma_{\mathrm{n}+1}$ yields: $\sigma_{\mathrm{n}+1}=\mathrm{E}\left(\varepsilon_{\mathrm{n}+1}-\right.$ $\varepsilon_{\mathrm{n}+1}^{\mathrm{vp}}$ ) which may be written:

$$
\sigma_{\mathrm{n}+1}=\mathrm{E}\left(\varepsilon_{\mathrm{n}+1}-\varepsilon_{\mathrm{n}}^{\mathrm{vp}}\right)-\mathrm{E}\left(\varepsilon_{\mathrm{n}+1}^{\mathrm{vp}}-\varepsilon_{\mathrm{n}}^{\mathrm{vp}}\right)
$$

thus: $\sigma_{\mathrm{n}+1}=\sigma_{\mathrm{n}+1}^{\mathrm{g}}-\mathrm{E} \Delta \gamma$ and the state of the system at time $t_{\mathrm{n}+1}$ is fully described by the following set of equations:

$$
\begin{aligned}
& \sigma_{\mathrm{n}+1}=\sigma_{\mathrm{n}+1}^{\mathrm{g}}-\mathrm{E} \Delta \gamma \\
& \varepsilon_{\mathrm{n}+1}^{v p}=\varepsilon_{\mathrm{n}}^{\mathrm{vp}}+\Delta \gamma \\
& \alpha_{\mathrm{n}+1}=\alpha_{\mathrm{n}}+\Delta \gamma \\
& f_{\mathrm{n}+1}=\sigma_{\mathrm{n}+1}-\left(\sigma_{\mathrm{Y}}+K \alpha_{\mathrm{n}+1}\right)
\end{aligned}
$$

where every term is known to the exception of $\Delta \gamma$. For the sake of brevity, details of the computation of this terms are not given here:

$$
\Delta \gamma=\frac{f_{\mathrm{n}+1}^{\mathrm{g}}+\gamma_{\mathrm{n}} \cdot \eta}{\frac{2}{h} \cdot \eta+\mathrm{E}+\mathrm{K}}
$$

and the system of equations given in Eq. (8) is now explicitly known.

The system is then fully described at the $(n+1)^{\text {th }}$ time step and the time step can be increased in order to proceed time integration until the end of the simulation is reached. 


\begin{tabular}{|c|c|}
\hline Numerical parameters & value \\
\hline $\mathrm{E}$ & $90 \cdot 10^{9} \mathrm{~Pa}$ \\
\hline $\mathrm{K}$ & $15 \cdot 10^{9} \mathrm{~Pa}$ \\
\hline$\eta$ & $5 \cdot 10^{4} \mathrm{~Pa} \cdot \mathrm{S}$ \\
\hline$\sigma_{Y}$ & $1 \cdot 10^{7} \mathrm{~Pa}$ \\
\hline$p$ & $\mathbf{2} \cdot \mathbf{1 0}^{-1} \mathrm{~mm}$ \\
\hline
\end{tabular}

Table 1 - Numerical parameters chosen for the sensitivity study.

\section{Tool/abradable material interaction}

The viscoplastic law used for the modeling of the abradable material involves four parameters: (1) the Young's modulus E, (2) the plastic modulus $K$, (3) the yield limit $\sigma_{Y}$, (4) the viscoplastic coefficient $\eta$ and (5) the penetration $p$ of the tool in the abradable material.

As mentioned above, our study intends to calibrate the parameters of the viscoplastic law in order to assess the relevance of the modeling and the feasibility of precise abradable material/tool simulations and thus more accurate blade/casing interaction simulations. While a good representation of abradable material removal - referred to as the wear level - is of great importance, the focus is essentially made on the tool side. Indeed, the critical aspect of a blade/casing interaction simulation definitely lies into the amplitude of vibrations of the blade and the subsequent stress level in it: through the prediction of stress level within the blade, designers may be able to predict and thus prevent cracks initiation.

Accordingly, our study does not intend to calibrate the viscoplastic law with material parameters close to the ones experimentally obtained for abradable material M601 [5]. Instead, it is expected to predict accurately the contact forces applied on the tool. This section features the description of an interaction simulation carried out with the numerical parameters given in Tab. 1, then the sensitivity of the model is assessed with respect to the five numerical parameters thus giving a better understanding of the role of each of these parameters and allowing for the calibration of the numerical model with experimental data.

\subsection{Interaction simulation}

The tool/abradable material interaction phenomenon occurs between times $t=t_{b}$ and $t=$ $t_{e}$. During the interaction, the focus is made on three quantities: (1) $\left(\mathrm{F}_{i}\right)_{i=1 . .5}$ the contact forces applied on the tool blade ${ }^{5}$ on each boundary node $\left(c_{i}\right)_{i=1 . .5}$ along the axial direction $\vec{z}$, (2) $\left(u_{x, i}\right)_{i=1 . .5}$ and $\left(u_{z, i}\right)_{i=1 . .5}$ the displacements of each boundary node $\left(c_{i}\right)_{i=1 . .5}$ respectively in the $\vec{x}$ and $\vec{z}$ directions and, (3) the wear profile that is obtained on the abradable material in front of each boundary node.

Even if the $r$ parameter depicted in Fig. 5 may be seen as the modeling of the keenness of the cutting edge of the tool, its numerical value is associated with numerical convergence considerations. The point being to avoid an all-or-nothing state in terms of penetration of

\footnotetext{
${ }^{5}$ As described in Eq. (1).
} 


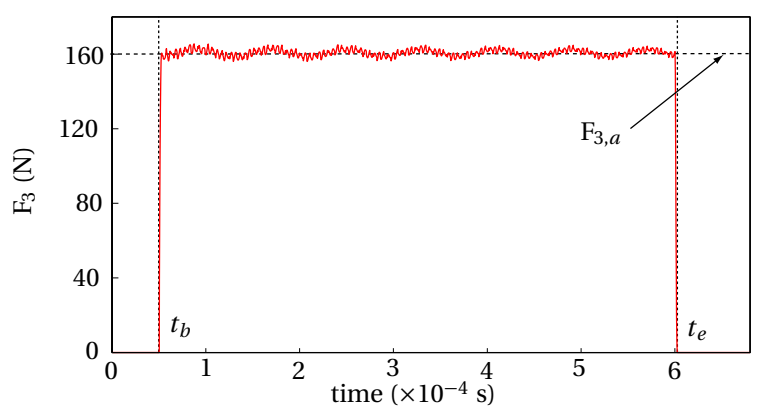

Figure 9 - Contact force applied on node $c_{3}$ during the interaction $\left(\|\overrightarrow{\mathrm{V}}\|=200 \mathrm{~m} \cdot \mathrm{s}^{-1}\right.$ and relative penetration $p=0.2 \mathrm{~mm}$ ).

the abradable in the tool at each time step which could lead to numerical instability. In our study, $r$ is set to $r=25 \mu \mathrm{m}$ which is consistent with the keenness of the cutting edge of the experimental tool.

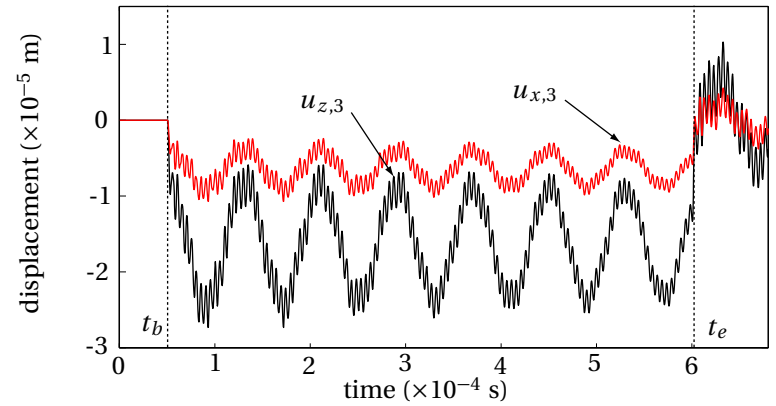

Figure 10 - Displacements $u_{x, 3}$ and $u_{z, 3}$ of contact node $c_{3}$ during the interaction $(\|\vec{V}\|=$ $200 \mathrm{~m} \cdot \mathrm{s}^{-1}$ and relative penetration $p=0.2 \mathrm{~mm}$ ).

In agreement with experiments detailed in the first section, the first simulation presented is carried out with a projectile speed $\|\overrightarrow{\mathrm{V}}\|=200 \mathrm{~m} \cdot \mathrm{s}^{-1}$ and a relative penetration $p=0.2 \mathrm{~mm}$. For the sake of clarity, contact forces and displacements respectively pictured in Fig. 9 and Fig. 10 are given for boundary node $c_{3}$ only. Very similar results may be observed on the other boundary nodes.

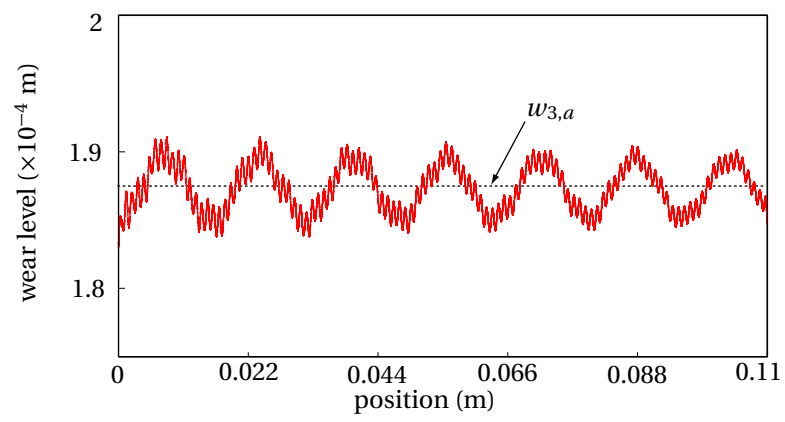

Figure 11 - Wear profile along the abradable material after the interaction $\left(\|\vec{V}\|=200 \mathrm{~m} \cdot \mathrm{s}^{-1}\right.$ and relative penetration $p=0.2 \mathrm{~mm}$ ).

As one may see in Fig. 9, the contact force on node $c_{3}$ in the $\vec{z}$ direction is almost constant during the interaction. An average value denoted $\mathrm{F}_{3, a}{ }^{6}$ is computed over $t \in\left[t_{b} ; t_{e}\right]$. The

${ }^{6}$ For a given contact node $k, \mathrm{~F}_{k, a}$ is the average value over time of the contact force $\mathrm{F}_{k}$ defined in Eq. (1). 
contact effort $\mathrm{F}_{3, a}$ is mostly dependent on the projectile speed $\overrightarrow{\mathrm{V}}$ and the penetration $p$. In the following sections, the axial contact force applied on the tool will be referred to as $\|\overrightarrow{\mathrm{F}}\|$ defined by:

$$
\|\overrightarrow{\mathrm{F}}\|=\sum_{k=1}^{5} \mathrm{~F}_{k, a}
$$

Displacements $u_{x, 3}$ and $u_{z, 3}$ are pictured in Fig. 10. Both displacements are negative during the interaction and one may see that their amplitudes is relatively small: about $2.5 \%$ of the relative penetration $p$ for $u_{x, 3}$.

Finally, the wear level on the abradable material resulting from the interaction with the tool is plot in Fig. 11. First, it is noticeable that the average value of the wear level - displayed as $w_{3, a}$ - is slightly less than the relative penetration $p: w_{3, a} \simeq 0.187 \mathrm{~mm}$ while $p=0.2 \mathrm{~mm}$. This is due to the elastic component of the deformation of the abradable elements during the interaction. Also, the amplitude of the lobes visible along this wear profile are in good agreement with the amplitude of the displacement $u_{x, 3}$ pictured in 10 .

\subsection{Sensitivity of the model}

This section aims at describing the impact of the variation of each numerical parameter (E, $\mathrm{K}, \eta, \sigma_{\mathrm{Y}}$ and $p$ ) of the viscoplastic law on the results. This sensitivity study is carried out around the set of initial values given in Tab. 1.

Results obtained with these values are plotted as a solid line in Figs. 12(a), 12(b), 12(c) and 12(d). For each figure, all the dashed lines are obtained with the variation of only one of the aforementioned numerical parameters.

Young's modulus variation from zero to infinite values describe a triangular area of admissible values as depicted in Fig. 12(a). When the Young's modulus tends to infinite, one may see that the relation between the axial force on the tool $\|\overrightarrow{\mathrm{F}}\|$ and the speed of the projectile becomes linear ${ }^{7}$.

Regarding the influence of the viscoplastic parameter $\eta$, one may see in Fig. 12(b) that if $\eta$ tends to zero, viscoplastic effects become negligible and the axial contact force becomes constant (low but positive), meaning independent from the speed of the projectile.

The increase of both the plastic modulus and the yield limit has a similar impact on the evolution of the results as depicted in Fig 12(c) and Fig. 12(d). Strongly increasing each of these parameters yield a positive offset value of the axial contact force when the speed of the tool is zero.

The last numerical parameter of interest is the penetration of the tool in the abradable

\footnotetext{
${ }^{7}$ Indeed, for extremely high values of the Young's modulus, the plastic modulus may be considered negligible thus plastic effects become negligible and the computation of the axial force on the tool solely involves the quantity of abradable material removed by the tool at each time step which is directly proportional to the projectile speed. In the present paper, plastic effects are not negligible and the liner is permanently deformed.
} 


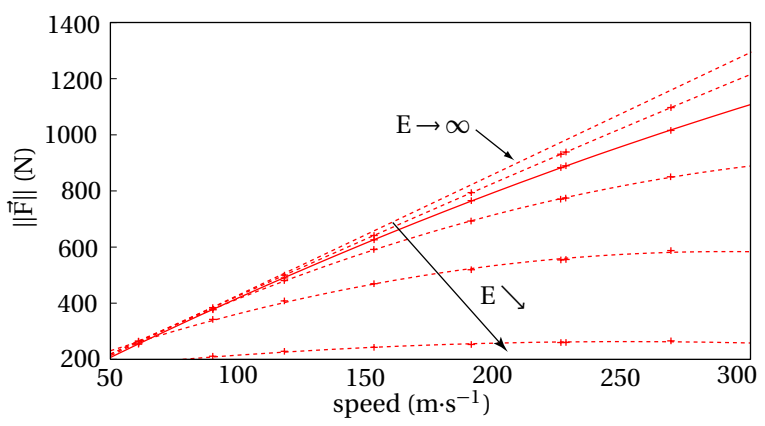

(a) Influence of Young's modulus.

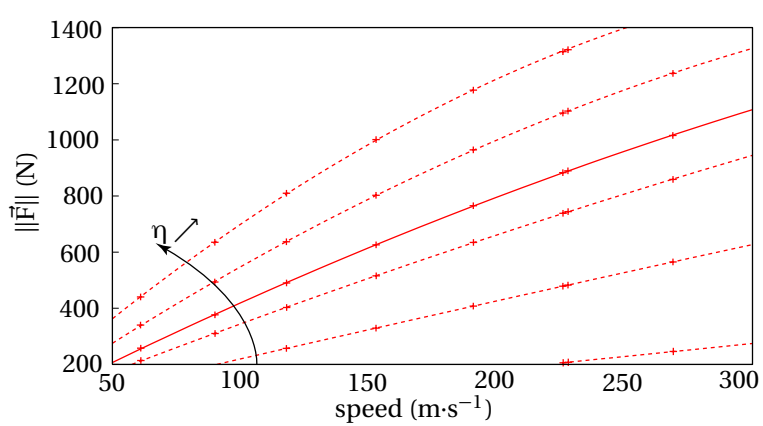

(b) Influence of the viscoplastic parameter $\eta$

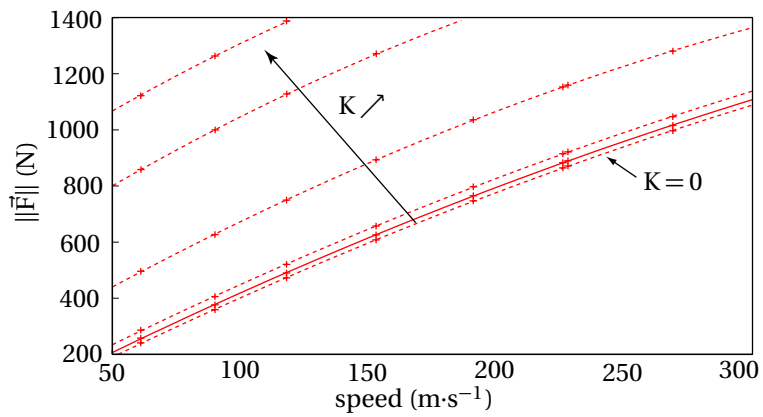

(c) Influence of the plastic modulus.

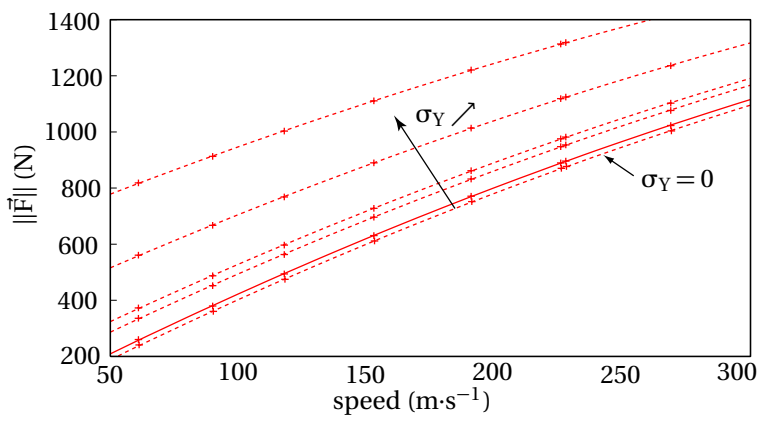

(d) Influence of the yield limit $\sigma_{Y}$.

Figure 12 - Evolution of the numerical results with respect to Young's modulus, the viscoplastic coefficient $\eta$, the plastic modulus $K$ and the yield limit $\sigma_{Y}$.

material $p$. Obviously, the higher $p$, the higher is the contact force on the blade and it is remarkable in Fig. 13 that the contact force varies very significantly for small variations of $p$. These results underline that any variation of the penetration experimentally observed should be taken into account in the numerical model.

Thanks to these observations, a calibration procedure allows to fit numerical results with experimental data. Calibrated parameters of the viscoplastic law are given in Tab. 2. One 


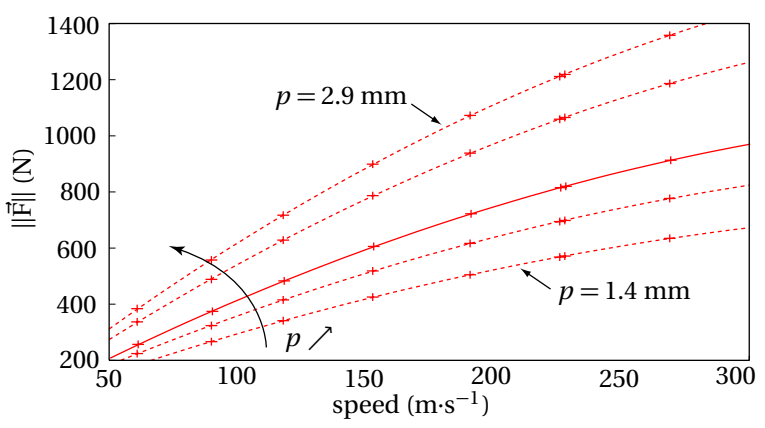

Figure 13 - Evolution of the numerical results with respect to the penetration of the tool in the abradable $p$.

may notice that the calibrated Young's modulus is significantly higher than the experimental value obtained for abradable material M601. It seems very likely that the modeling of abrad-

\begin{tabular}{ccc}
\hline Numerical parameter & Calibrated value & $\begin{array}{c}\text { Experimental value [5] } \\
\text { (static values) }\end{array}$ \\
\hline $\mathrm{E}$ & $\mathbf{6 0} \cdot \mathbf{1 0}^{\mathbf{9}} \mathrm{Pa}$ & $2.1 \pm 0.9 \cdot 10^{9} \mathrm{~Pa}$ \\
$\mathrm{~K}$ & $\mathbf{1 5}^{\mathbf{9}} \mathrm{\mathbf {10 } ^ { 9 }} \mathrm{Pa}$ & - \\
$\eta$ & $\mathbf{5} \cdot \mathbf{1 0}^{4} \mathrm{~Pa} \cdot \mathrm{s}$ & - \\
$\sigma_{\mathrm{Y}}$ & $\mathbf{7 \cdot \mathbf { 1 0 } ^ { 6 } \mathrm { Pa }}$ & $6.9 \cdot 10^{6} \mathrm{~Pa}$ \\
\hline
\end{tabular}

Table 2 - Numerical and experimental material properties of the abradable material.

able material removal through the compression of one-dimensional abradable elements is responsible for this numerical over-evaluation of the abradable material mechanical properties.

\subsection{Confrontation with experimental observations}

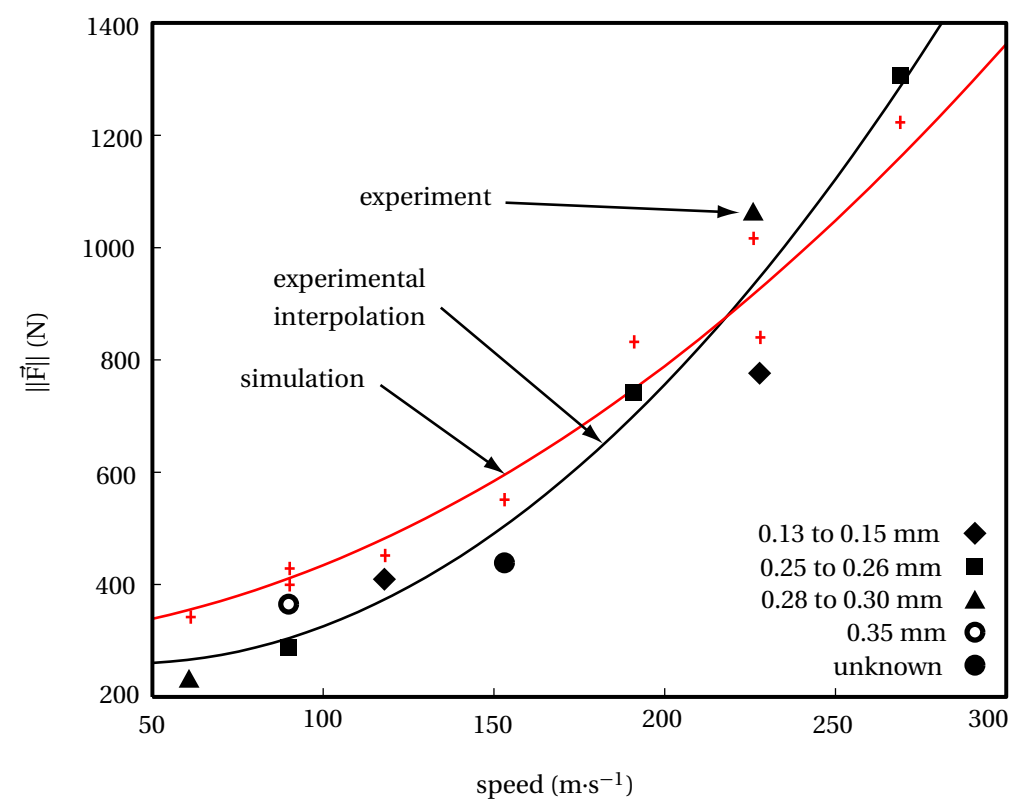

Figure 14 - Superimposition of experimental data and numerical results with consistent penetration values $p$ between numerical simulations and experimental observations.

The penetration in the abradable is experimentally set to $p=0.2 \mathrm{~mm}$. Because of the large interaction speed range covered - from 50 to $250 \mathrm{~m} \cdot \mathrm{s}^{-1}$ - this data set may be compared 
with an actual blade-tip/abradable coating interaction case.

Numerical simulations are launched taking into account the variation of the experimental penetration observed for each point. Superimposition of the results of these numerical simulations with experimental results is depicted in Fig. 14. The curvatures of both results interpolations are consistent and numerical results match very well with experimental measurements.

Results show that the proposed strategy is well suited for the modeling of the tool/abradable material interaction phenomenon: when the experimental variation of the penetration is taken into account ${ }^{8}$ both the amplitude of the axial contact force and its evolution with respect to the projectile speed are accurately reproduced.

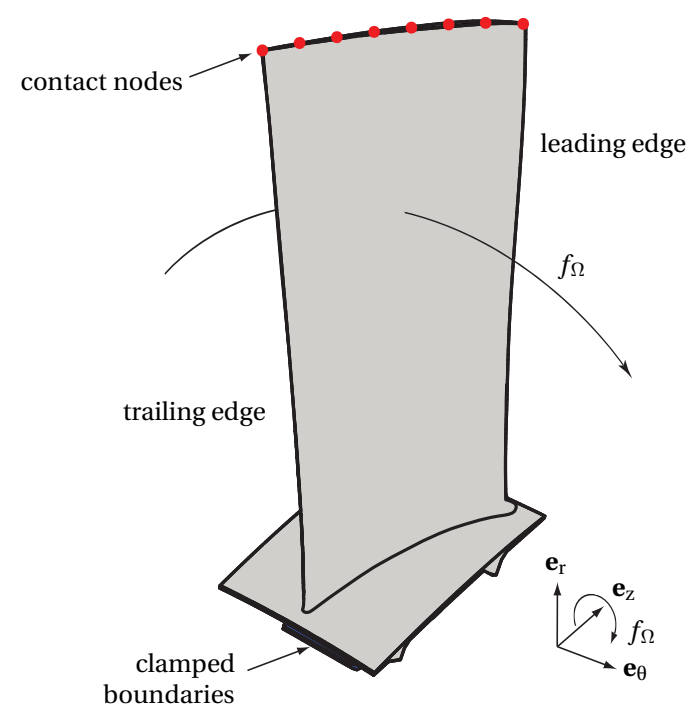

Figure 15 - Blade model.

A more precise calibration of the numerical simulation may be obtained but would not be relevant here due to the relatively few available experimental data. In particular, it would be of great interest to experimentally assess the dispersion around each measured point in order to maximize the precision of the interpolation line of the experimental results.

\section{Application to turbomachines}

For the sake of confidentiality, all the frequencies given in this section are normalized with respect to the first eigenfrequency $f_{1}$ of the blade.

The simulation of the tool/abradable material interaction suggests the need for a significant evolution of the numerical strategy proposed in [6] through the implementation of a viscoplastic law in order to take into account the strain-rate dependence.

Though, when speaking of blade-tip/abradable coating interaction, one may question

\footnotetext{
${ }^{8}$ The point for which no penetration data is available is not considered for the interpolation of numerical and experimental results.
} 


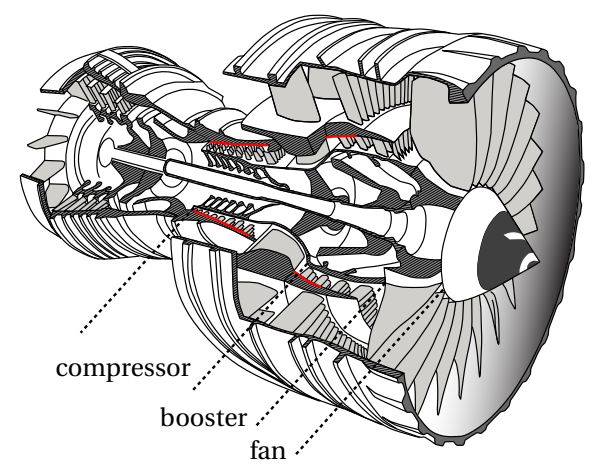

Figure 16 - Partial cut view of a turbomachine with sensitive contact areas ( -$)$.

the need for the viscoplastic law. Indeed, strain-rates $\dot{\varepsilon}_{\text {exp }}$ observed experimentally are very distinct from those usually observed in turbomachines $\dot{\varepsilon}_{\text {tur. }}$ The imposed penetration $p$ of the tool within the abradable material yields $\dot{\varepsilon}_{\exp }>10^{3} \mathrm{~s}^{-1}$ (numerical simulations give $\dot{\varepsilon}_{\text {exp }} \simeq 2 \cdot 10^{4} \mathrm{~s}^{-1}$ ) while usual blade-tip/abradable coating penetrations lead roughly to $\dot{\varepsilon}_{\text {turb }} \simeq$ $10^{1} \mathrm{~s}^{-1}$ (numerical simulations give $5 \leq \dot{\varepsilon}_{\text {turb }} \leq 25$ ).

Accordingly, this section aims at assessing the role of parameter $\eta$ in the simulation of blade-tip/abradable coating interactions.

Blade-tip/casing contact areas of interest are depicted in Fig. 16. The blade model used for the simulations is the same as the one described in [6] and is depicted in Fig. 15.

Numerical simulation of the interaction phenomenon between blades and the abradable coating could allow for the identification of critical stress levels areas within a blade or to the comparison of blade responses when different designs are considered.

An explicit time integration scheme is combined with a reduced order model of a blade of one stage of the turbomachine and centrifugal stiffening is taken into account. The abradable modeling is updated since the plastic law is replaced by the proposed viscoplastic law detailed in this paper. Simulations are launched with the mechanical properties of the abradable material given in Tab. 2.

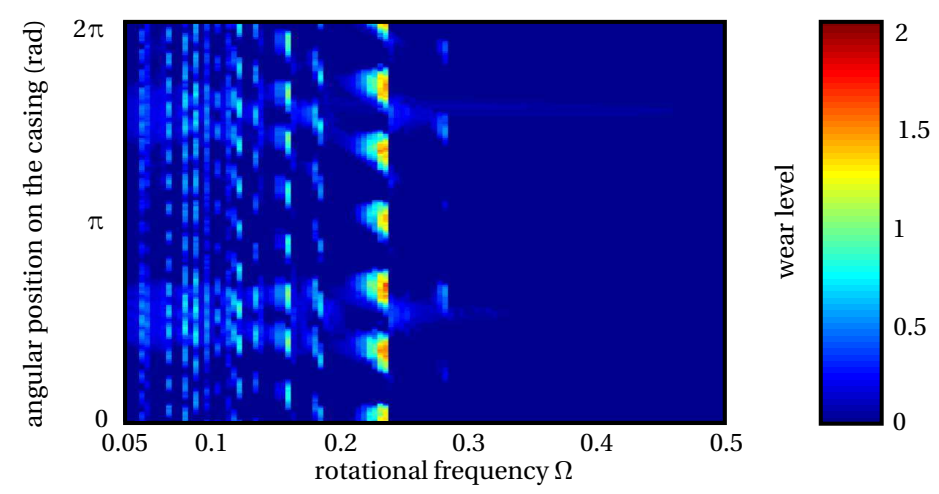

Figure 17 - Maps of the final abradable profiles on the trailing edge with respect to $\Omega$ after fifty rounds of the blade with $\eta=0$ Pa.s

Regarding the contact configuration, the casing - assumed perfectly rigid - is distorted 
along a combination of a 2- and 3-nodal diameter free vibration modes. The resulting maximal radial displacement is about twice the initial blade-tip/abradable clearance ${ }^{9}$ at $f_{\Omega}=0$. Simulation time is chosen in such a way that the blade makes exactly fifty turns and it takes about fifteen turns to reach steady state after contact initiation during the first revolution of the blade.

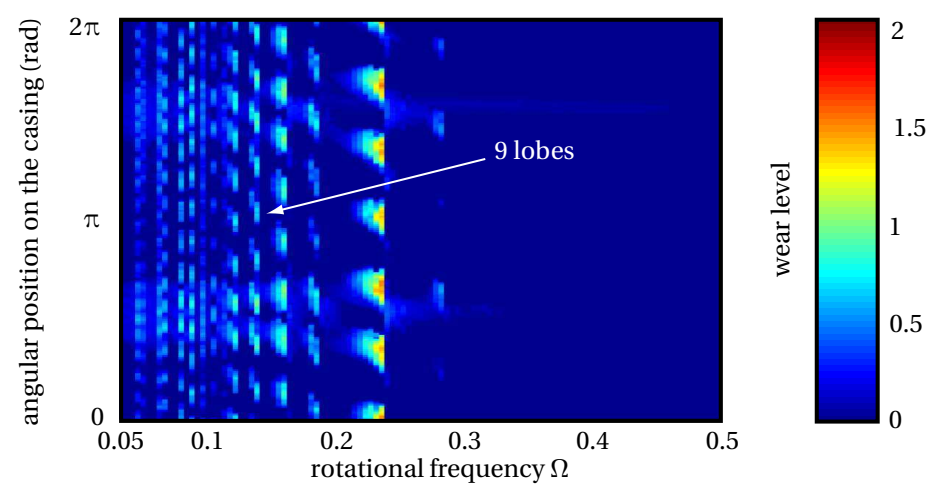

Figure 18 - Maps of the final abradable profiles on the trailing edge with respect to $\Omega$ after fifty rounds of the blade with $\eta=50,000 \mathrm{~Pa} \cdot \mathrm{s}$

For each rotational frequency, the final wear profile of the abradable material is observed with respect to the angular position on the casing. Put side-by-side, these profiles create a wear map useful for an identification of critical velocities where the penetration of the blade in the abradable may reach critical values.

Wear maps are plot for the trailing edge for $\eta=0$ Pa.s in Fig. 17 and for $\eta=50,000$ Pa.s in Fig. 18.

No major difference appears between these two maps. One may notice that, globally, wear levels are slightly higher when $\eta=50,000 \mathrm{~Pa} \cdot \mathrm{s}$. At first, this result may be surprising considering that the yield limit depends on $\eta$ and the higher it is, the higher must be the stress in the abradable element to reach plastic deformation. However, previous results [7] highlighted that stiffer abradable materials could lead to higher level of vibrations and subsequent higher wear levels.

This slight increase of wear level may locally lead to new interaction profiles such as the 9-lobe wear profile - shown in Fig. 18 - that appears for $\eta=50,000$ Pa.s and $f_{\Omega}=0.13$. However, these interaction profiles feature relatively low vibration amplitude levels in comparison with the main interaction rotational frequencies $\left(f_{\Omega}=0.23\right.$ and $f_{\Omega}=0.286$ ) and thus do not significantly impact the dynamics of the blade.

Accordingly, the results given in this section confirm that $\eta$ parameter has a negligible influence over the simulation of the blade-tip/abradable coating interaction simulation and that the plasticity law detailed in [6] may be used without significant loss of accuracy.

${ }^{9}$ Centrifugal effects being taken into account, the initial blade-tip/abradable clearance depends on the rotational frequency of the blade. 


\section{Conclusion}

Based on the numerical strategy proposed in [6], a viscoplastic law is developed and applied for the numerical simulation of tool/abradable material interaction simulation. The material parameters of this law are calibrated with respect to experimental measurements and it is shown that numerical and experimental results match very well over the range of interaction speeds considered. Both the amplitude of the contact force applied on the tool and its evolution with respect to the projectile speed are accurately reproduced. Thus, the proposed numerical approach seems well suited for modeling the tool/abradable material interaction phenomenon.

In order to assess the influence of the viscoplastic law for blade-tip/abradable coating interaction simulations, an application case is detailed where results obtained with the new viscoplastic law are compared with those obtained with the initial plastic law. It is confirmed that, because of the relatively small strain-rates encountered for such interactions, the influence of the viscoplastic coefficient on the results is negligible.

\section{Acknowledgement}

Thanks go to Snecma for its technical and financial support. This work takes place in the framework of the MAIA mechanical research and technology program sponsored by CNRS, ONERA and SAFRAN Group.

\section{References}

[1] M. Legrand, C. Pierre, P. Cartraud, and J.-P. Lombard. Two-dimensional modeling of an aircraft engine structural bladed disk-casing modal interaction. Journal of Sound and Vibration, 319(1-2):366 - 391, 2009. ISSN 0022-460X. doi: 10.1016/j.jsv.2008.06.019. DOI:10.1016/j.jsv.2008.06.019 also available on HAL:hal-00328186.

[2] G. V. Groll and Ewins D. J. The harmonic balance method with arc-length continuation in rotor/stator contact problems. Journal of Sound and Vibration, 241:223-233, 2001. DOI:10.1006/jsvi.2000.3298.

[3] C. Padova, J. Barton, M.G. Dunn, S. Manwaring, G. Young, M.Jr. Adams, and M. Adams. Development of an experimental capability to produce controlled blade tip/shroud rubs at engine speed. Journal of Turbomachinery, 127:726-735, 2005.

[4] M. Yi, He J., B. Huang, and H. Zhou. Friction and wear behaviour and abradability of abradable seal coating. Wear, 231:47-53, 1999. 
[5] X. Ma and A. Matthews. Evaluation of abradable seal coating mechanical properties. Wear, 267:1501-1510, 2009. DOI:10.1016/j.wear.2009.03.044.

[6] M. Legrand, A. Batailly, and C. Pierre. Numerical investigation of abradable coating removal in aircraft engines through plastic constitutive law. Journal of Computational and Nonlinear Dynamics, 7, 2011. doi: 10.1115/1.4004951. DOI:10.1115/1.4004951 and available on HAL:hal-00627526.

[7] A. Batailly, M. Legrand, and C. Pierre. Influence of abradable coating wear mechanical properties on rotor stator interaction. In Proceedings of the ASME Turbo Expo 2011 GT2011-45189, Vancouver, Canada, 2008. available on HAL:hal-00616524_v1.

[8] F. Peyraut, J. L. Seichepine, C. Coddet, and M. Hertter. Finite element modeling of abradable materials - identification of plastic parameters and issues on minimum hardness against coating's thickness. International Journal for Simulation and Multidisciplinary Design Optimization, 2:209-215, 2008. DOI:10.1051/ijsmdo:2008028.

[9] M. Bounazef, S. Guessasma, and Ait Saadi B. The wear, deterioration and transformation phenomena of abradable coating BN-SiAl-bounding organic element, caused by the friction between the blades and the turbine casing. materials letters, 58:3375-3380, 2004. DOI:10.1016/j.matlet.2004.02.049.

[10] M. Yi, He J., B. Huang, and H. Zhou. Abradability evaluation and tribological behaviour of abradable seal coating. Trans. Nonferrous Met. Soc. China, 8:459-467, 1998.

[11] R.R. Craig and C.C. Bampton. Coupling of substructures for dynamics analyses. AIAA Journal, 6(7):1313 - 1319, 1968. doi: 10.2514/3.4741.

[12] N. D. Cristescu. Dynamic plasticity. 1967.

[13] J. C. Simo and T. J. R. Hughes. Computational inelasticity. Springer-Verlag, 1998.

[14] V. I. Zubov, G. V. Stepanov, and Shirokov A. V. Effect of the strain rate on the yield strength of steels of different strength. Strength of Materials, 35:514-520, 2003. 